\title{
CONVERGENCE TO ENDS FOR RANDOM WALKS ON THE AUTOMORPHISM GROUP OF A TREE
}

\author{
DONALD I. CARTWRIGHT AND P. M. SOARDI
}

(Communicated by William D. Sudderth)

\begin{abstract}
Let $\mu$ be a probability on a free group $\Gamma$ of rank $r \geq 2$. Assume that $\operatorname{Supp}(\mu)$ is not contained in a cyclic subgroup of $\Gamma$. We show that if $\left(X_{n}\right)_{n \geq 0}$ is the right random walk on $\Gamma$ determined by $\mu$, then with probability $1, X_{n}$ converges (in the natural sense) to an infinite reduced word. The space $\Omega$ of infinite reduced words carries a unique probability $\nu$ such that $(\Omega, \nu)$ is a frontier of $(\Gamma, \mu)$ in the sense of Furstenberg [10]. This result extends to the right random walk $\left(X_{n}\right)$ determined by a probability $\mu$ on the group $G$ of automorphisms of an arbitrary infinite locally finite tree $T$. Assuming that $\operatorname{Supp}(\mu)$ is not contained in any amenable closed subgroup of $G$, then with probability 1 there is an end $\omega$ of $T$ such that $X_{n} v$ converges to $\omega$ for each $v \in T$. Our methods are principally drawn from [9] and [10].
\end{abstract}

\section{INTRODUCTION AND PRELIMINARIES}

Let $\mu$ be a probability on the free group $\Gamma$ generated by $\left\{a_{1}, \ldots, a_{r}\right\}$, where $r \geq 2$. Consider the right random walk $\left(X_{n}\right)_{n \geq 0}$ on $\Gamma$ determined by $\mu$; i.e., $X_{0}=e$, and $X_{n}=Y_{1} Y_{2} \cdots Y_{n}$ for $n \geq 1$, where the $Y_{j}$ 's are independent $\Gamma$-valued random variables on some probability space $(\Xi, \mathscr{F}, P)$, with $P\left(Y_{j}=x\right)=\mu(x)$ for each $x \in \Gamma$ and $j \geq 1$. Thus $\left(X_{n}\right)_{n \geq 0}$ is a Markov chain with transition probabilities $p(x, y)=\mu\left(x^{-1} y\right)$. It is well known that this chain is transient provided that it is irreducible, i.e., provided that for each $x \in \Gamma$ there is an $n \geq 1$ so that $\mu^{* n}(x)=P\left(X_{n}=x \mid X_{0}=e\right)>0$. In fact (see [1, p. 43] or [7]), it is transient provided that:

The support $\operatorname{Supp}(\mu)$ of $\mu$ is not contained in a cyclic subgroup of $\Gamma$.

Indeed, every noncyclic subgroup of $\Gamma$ is nonamenable, by Schreier's Theorem [14]. Each $x \in \Gamma$ can be written in a unique way as a reduced word $x=$ $x_{1} x_{2} \cdots x_{m}$, where $m \geq 0$, each $x_{i}$ is an $a_{j}$ or $a_{j}^{-1}$, and $x_{i+1} \neq x_{i}^{-1}$ for all $i<m$; we write $|x|=m$. Transience of $\left(X_{n}\right)$ just means that $\left|X_{n}\right| \rightarrow \infty$ with

Received by the editors November 23, 1988

1980 Mathematics Subject Classification (1985 Revision). Primary 60J15, 60J50; Secondary $05 \mathrm{C} 05,43 \mathrm{~A} 05$.

Key words and phrases. Random walks, free groups, ends of trees, boundaries of groups. 
probability 1. A finer description of this transience is obtained by considering the space $\Omega$ of infinite reduced words $\omega=x_{1} x_{2} \cdots x_{m} \cdots$, where each $x_{i}$ is an $a_{j}$ or $a_{j}^{-1}$ and $x_{i+1} \neq x_{i}^{-1}$ for all $i$. It will follow from our main result (see Corollary 2.3, page 821) that assuming (1.1), with probability $1, X_{n}$ converges to an $\omega$, i.e., for each $r \geq 1$ there is an $n_{r}$ so that once $n \geq n_{r}$, the first $r$ letters of the reduced word expression of $X_{n}$ agree with the first $r$ letters of $\omega$ (here $\omega$ depends on the point $\xi$ of the underlying probability space). This result has been proved under more restrictive conditions on $\mu$ in [6] (where $\operatorname{Supp}(\mu)$ is finite), [13] (where $\left.\sum_{x \in \Gamma} \log (|x|+1) \mu(x)<\infty\right)$, [5] ( where $\mu(x)$ depends only on $|x|$ ), and it is attributed (without proof) in [11] to G. A. Margulis (assuming only irreducibility?).

It follows from our result that $\Omega$, together with a measure $\nu$ it carries, uniquely determined by $\mu$, is a frontier for $(\Gamma, \mu)$ in the sense of [10].

Now let $T$ be an infinite locally finite tree (see, e.g., [14]). Let $G$ denote the group of automorphisms of $T$, i.e., mappings of $T$ onto itself which preserve the natural distance $d$ on $T$. Equipped with the topology of pointwise convergence, $G$ is a totally disconnected locally compact group. The subgroups $G(F)=\{\varphi \in G: \varphi(v)=v$ for all $v \in F\}$, where $F \subset T$ is finite, form a base of compact open neighbourhoods of the identity in $G$ (see, e.g., [15]). As in [4], the ends of $T$ are equivalence classes $\omega$ of infinite chains, i.e., sequences $\left(v_{0}, v_{1}, \ldots\right)$ of distinct vertices of $T$, in which $v_{i}$ is a neighbour of $v_{i+1}$ for each $i$. Chains $\left(v_{0}, v_{1}, \ldots\right)$ and $\left(v_{0}^{\prime}, v_{1}^{\prime}, \ldots\right)$ are equivalent if there exist $r$ and $s$ so that $v_{r+i}=v_{s+i}^{\prime}$ for all $i \geq 0$. If we fix a vertex $v_{0}$ in $T$, then each end $\omega$ contains exactly one chain starting from $v_{0}$, which we call the geodesic from $v_{0}$ to $\omega$. Let $\Omega$ denote the set of ends. There is a natural topology on $T \cup \Omega$, making it a compact Hausdorff space containing $T$ as a discrete dense subset. We may describe this topology as follows. Fix $v_{0} \in T$. If $\omega \neq \omega^{\prime} \in \Omega$, let $\left(v_{0}, v_{1}, \ldots\right)$ and $\left(v_{0}^{\prime}=v_{0}, v_{1}^{\prime}, \ldots\right)$ be the geodesics from $v_{0}$ to $\omega$ and $\omega^{\prime}$ respectively. Let $j\left(\omega, \omega^{\prime}\right)$ be the largest $j \geq 0$ such that $v_{j}=v_{j}^{\prime}$, and set $j(\omega, \omega)=\infty$. We may similarly define $j(v, \omega)$ and $j\left(v, v^{\prime}\right)$ for $v, v^{\prime} \in T$ and $\omega \in \Omega$. Then the sets $N_{k}=\left\{\omega \in T \cup \Omega: j\left(\omega, \omega_{0}\right) \geq k\right\}, k=1,2, \ldots$, form a base of neighbourhoods of $\omega_{0} \in \Omega$. Notice that $j\left(\omega, \omega^{\prime}\right)$ depends on $v_{0}$, but the topology does not. If $\omega \in \Omega$ and $\varphi \in G$, then $\varphi \omega$ is defined to be the end of the chain $\left(\varphi\left(v_{0}\right), \varphi\left(v_{1}\right), \ldots\right)$, where $\left(v_{0}, v_{1}, \ldots\right)$ is the geodesic from $v_{0}$ to $\omega$. This defines a continuous action of $G$ on $\Omega$.

Now let $\mu$ denote a probability measure on $G$ and let $Y_{1}, Y_{2}, \ldots, Y_{n}, \ldots$ denote $G$-valued i.i.d. random variables with common distribution $\mu$. Thus $X_{0}=e, X_{n}=Y_{1} Y_{2} \cdots Y_{n}$ is a right random walk on $G$ with transition probabilities $p(x, B)=\mu\left(x^{-1} B\right)$ for all $x \in G$ and all Borel sets $B \subseteq G$. Since $T$ is a $G$-space (in the sense of Furstenberg) we may consider the process $X_{n}\left(v_{0}\right)$ where $v_{0}$ is any fixed element of $T$. 
The main result of this paper consists in proving that if the support of $\mu$ is not contained in any amenable subgroup of $G$ then, with probability 1 , there exists an end $\omega \in \Omega$ such that $X_{n}\left(v_{0}\right) \rightarrow \omega$ and $n \rightarrow \infty$ for every $v_{0} \in T$.

The free group $\Gamma$ becomes a homogeneous tree of degree $2 r$ if for each $x \in \Gamma$ an edge is drawn between $x$ and the $2 r$ elements $x a_{j}^{ \pm 1}$ (the Cayley graph). Left multiplication then provides a natural embedding of $\Gamma$ as a discrete subgroup of the group of automorphisms of this tree. Taking $v_{0}=e$ we may identify ends with infinite reduced words. When $\varphi=g \in \Gamma, \varphi \omega$ is then obtained by multiplying the infinite reduced word $\omega$ on the left by $g$ and cancelling where possible.

\section{Statements AND PROOFS OF Results}

Let $v_{0}$ be a fixed vertex of $T$, and write $|v|$ for $d\left(v_{0}, v\right)$.

Lemma 2.1. If $\operatorname{Supp}(\mu)$ is not contained in a compact subgroup of $G$, then with probability 1 the sequence $\left(\left|X_{n}\left(v_{0}\right)\right|\right)$ is unbounded.

Proof. As usual, we may suppose that $(\Xi, \mathscr{F}, P)=\left(G^{\mathbf{N}}, \mathscr{B}_{G}^{\mathbf{N}}, \mu^{\mathbf{N}}\right)$ and the $Y_{j}$ 's are the coordinate functions (use, e.g., [2, Prop . 2.39]). The left shift $t$ on $\Xi$ is measure-preserving and ergodic. Now $\Xi^{\prime}=\left\{\xi \in \Xi:\left(\left|X_{n}\left(v_{0}\right)\right|\right)\right.$ is bounded $\}$ is a $t$-invariant set, and so $P\left(\Xi^{\prime}\right)=0$ or 1 . Suppose that $P\left(\Xi^{\prime}\right)=1$. Let $U$ be a compact neighbourhood of 1 in $G$. Let $S$ be a countable dense subset of $\operatorname{Supp}(\mu)$. Then for each $m \geq 1$ and each $\left(g_{1}, \ldots, g_{m}\right) \in S^{m}$, let $V$ be a neighbourhood of 1 such that $g_{1} V g_{2} V \cdots g_{m} V \subset g_{1} g_{2} \cdots g_{m} U$. Let

$$
A=\left\{\xi=\left(y_{1}, y_{2}, \ldots\right) \in \Xi: y_{j} \in g_{j} V \text { for } j=1, \ldots, m\right\} .
$$

Then $P(A)=\prod_{j=1}^{m} \mu\left(g_{j} V\right)>0$. By the Ergodic Theorem,

$$
\frac{1}{n} \sum_{k=0}^{n-1} \chi_{A}\left(t^{k} \xi\right) \rightarrow P(A)
$$

with probability 1 , say for $\xi \in \Xi_{m, g_{1}, \ldots, g_{m}}$. Let

$$
\Xi^{\prime \prime}=\bigcap_{m \geq 1, g_{1}, \ldots, g_{m} \in S} \Xi_{m, g_{1}, \ldots, g_{m}} \text {. }
$$

Then $P\left(\Xi^{\prime \prime}\right)=1$. Now let $\xi \in \Xi^{\prime} \cap \Xi^{\prime \prime}$. Since $\xi \in \Xi^{\prime}$, there is a compact set $K=K_{\xi} \subset G$ such that $X_{n}(\xi) \in K$ for all $n$. If $m \geq 1$ and $g_{1}, \ldots, g_{m} \in S$, then (2.1) implies that $t^{k} \xi \in A$ infinitely often, and so $Y_{k+1}(\xi) \cdots Y_{k+m}(\xi) \in$ $g_{1} \cdots g_{m} U$ for infinitely many $k$. Hence $Y_{k+1}(\xi) \cdots Y_{k+m}(\xi) \in K^{-1} K \cap$ $g_{1} \cdots g_{m} U$, so that $g_{1} \cdots g_{m} \in K^{-1} K U^{-1}$. This implies that the closed semigroup generated by $\operatorname{Supp}(\mu)$ is compact, and hence is a group [3]. This contradiction to our hypothesis shows that $P\left(\Xi^{\prime}\right)=0$.

Lemma 2.2. Let $\left(\varphi_{n}\right)$ be a sequence in $G$ such that $\varphi_{n}\left(v_{0}\right) \rightarrow \omega_{0} \in \Omega$ and $\varphi_{n}^{-1}\left(v_{0}\right) \rightarrow \omega^{*} \in \Omega$. Then $\varphi_{n} \omega \rightarrow \omega_{0}$ for each $\omega \in(T \cup \Omega) \backslash\left\{\omega^{*}\right\}$. 
Proof. Let us first observe that if $\varphi \in G, v \in T$ and $j\left(v, \varphi^{-1}\left(v_{0}\right)\right)=k<m=$ $\left|\varphi\left(v_{0}\right)\right|=\left|\varphi^{-1}\left(v_{0}\right)\right|$, then $j\left(\varphi(v), \varphi\left(v_{0}\right)\right)=m-k$. Because if $\left(v_{0}, v_{1}, \cdots, v_{m}=\varphi^{-1}\left(v_{0}\right)\right)$ and $\left(w_{0}=v_{0}, w_{1}, \ldots, w_{r}=v\right)$ are the geodesics from $v_{0}$ to $\varphi^{-1}\left(v_{0}\right)$ and $v$ respectively, then $v_{j}=w_{j}$ for $j \leq k$, while $v_{k+1} \neq w_{k+1}$. Then

$$
\left(v_{0}=\varphi\left(v_{m}\right), \varphi\left(v_{m-1}\right), \ldots, \varphi\left(v_{k}\right), \varphi\left(w_{k+1}\right), \ldots, \varphi\left(w_{r}\right)=\varphi(v)\right)
$$

is the geodesic from $v_{0}$ to $\varphi(v)$, while $\left(v_{0}=\varphi\left(v_{m}\right), \ldots, \varphi\left(v_{j}\right), \ldots, \varphi\left(v_{0}\right)\right)$ is that from $v_{0}$ to $\varphi\left(v_{0}\right)$.

Now let $\omega \in(T \cup \Omega) \backslash\left\{\omega^{*}\right\}$. Let $\left(v_{0}, v_{1}, \ldots\right)$ be the geodesic from $v_{0}$ to $\omega$. Then $j=j\left(\omega, \omega^{*}\right)<\infty$. Once $j\left(\varphi_{n}^{-1}\left(v_{0}\right), \omega^{*}\right)>j$, we have $j\left(\varphi_{n}^{-1}\left(v_{0}\right), v_{k}\right) \leq$ $j$ for all $k$. Hence, by the above, $j\left(\varphi_{n}\left(v_{k}\right), \varphi_{n}\left(v_{0}\right)\right) \geq\left|\varphi_{n}\left(v_{0}\right)\right|-j$. Thus given $N$, once $j\left(\varphi_{n}\left(v_{0}\right), \omega_{0}\right) \geq N, j\left(\varphi_{n}^{-1}\left(v_{0}\right), \omega^{*}\right)>j$ and $\left|\varphi_{n}\left(v_{0}\right)\right| \geq N+j$, we have $j\left(\varphi_{n}\left(v_{k}\right), \omega_{0}\right) \geq N$ for all $k$, so that $j\left(\varphi_{n} \omega, \omega_{0}\right) \geq N$.

Theorem. Let $\mu$ be a regular Borel probability on the group $G$ of automorphisms of a locally finite infinite tree $T$. Let $\left(X_{n}\right)_{n \geq 0}$ be a corresponding right random walk on $G$. Assume that $\operatorname{Supp}(\mu)$ is not contained in any amenable subgroup of $G$. Then with probability 1 there is an end $\omega \in \Omega$ such that $X_{n}(v) \rightarrow \omega$ in $T \cup \Omega$ for each fixed $v \in T$.

Proof. Consider the induced action of $G$ on $\Omega$. Since $\Omega$ is compact, there is a probability $\nu$ on $\Omega$ such that $\mu * \nu=\nu$, i.e.,

$$
\int_{\Omega} f(\omega) d \nu(\omega)=\int_{\Omega} \int_{G} f(g \omega) d \mu(g) d \nu(\omega)
$$

for all continuous $f$ on $\Omega$. The nonamenability hypothesis implies that $\nu$ is a continuous measure. For if $\nu(\{\omega\})>0$ for some $\omega \in \Omega$, let $a=$ $\max \{\nu(\{\omega\}): \omega \in \Omega\}$ and $S=\{\omega \in \Omega: \nu(\{\omega\})=a\}$. Then $S$ is finite, and $g S=S$ for all $g \in \operatorname{Supp}(\mu)$ because $\mu * \nu=\nu$. But Nebbia [12] showed that if $\omega_{0} \in \Omega$, then the closed group $G_{\omega_{0}}=\left\{g \in G: g \omega_{0}=\omega_{o}\right\}$ is amenable. Thus if $\omega_{o} \in S$, then $G_{S}=\{g \in G: g \omega=\omega$ for all $\omega \in S\}$ is a closed subgroup of $G_{\omega_{0}}$, and hence amenable. But $H=\{g \in G: g S=S\}$ contains $G_{S}$ as a normal subgroup of finite index, and is therefore amenable too. So $\operatorname{Supp}(\mu) \subset H$ is contrary to our hypothesis.

According to [10, Lemma 3.1(Corollary)], with probability $1, X_{n} \nu$ converges weak*. That is, there is a set $N \subset \Xi$ with $P(N)=0$, so that if $\xi \in \Xi \backslash N$, there is a probability $\lambda_{\xi}$ on $\Omega$ so that

$$
\int_{\Omega} f\left(X_{n}(\xi) \omega\right) d \nu(\omega) \rightarrow \int_{\Omega} f(\omega) d \lambda_{\xi}(\omega)
$$

for each $f$ in the space $\mathscr{C}(\Omega)$ of continuous functions on $\Omega$. We shall show that with probability $1, \lambda_{\xi}$ is a Dirac measure $\delta_{\omega_{0}(\xi)}$.

By Lemma 2.1, there is a null set $N_{1}$ so that $\left(\left|X_{n}(\xi) v_{0}\right|\right)$ is unbounded if $\xi \notin N_{1}$. Fix $\xi \notin N \cup N_{1}$. Let $\omega_{0} \in \Omega$ be a cluster point of $\left(X_{n}(\xi) v_{0}\right)$. Then 
we may choose a subsequence $\left(\varphi_{j}\right)$ of $\left(X_{n}(\xi)\right)$ such that $\varphi_{j}\left(v_{0}\right) \rightarrow \omega_{0}$ and $\varphi_{j}^{-1}\left(v_{0}\right) \rightarrow \omega^{*}$ for some $\omega^{*} \in \Omega$. Then $\varphi_{j} \omega \rightarrow \omega_{0}$ for all $\omega \in \Omega \backslash\left\{\omega^{*}\right\}$ by Lemma 2.2. But

$$
\int_{\Omega} f\left(\varphi_{j} \omega\right) d \nu(\omega) \rightarrow \int_{\Omega} f(\omega) d \lambda_{\xi}(\omega)
$$

for all $f \in \mathscr{C}(\Omega)$, and $\nu\left\{\omega^{*}\right\}=0$. Hence $\lambda_{\xi}=\delta_{\omega_{0}}$. This shows that $\omega_{0}$ is the only cluster point of $\left(X_{n}(\xi) v_{0}\right)$ in $\Omega$. Once we have checked that $\left|X_{n}(\xi) v_{0}\right| \rightarrow$ $\infty$, it will then follow that $X_{n}(\xi) v_{0} \rightarrow \omega_{0}$, and hence that $X_{n}(\xi) v \rightarrow \omega_{0}$ for all $v \in T$. But if $\left|X_{n}(\xi) v_{0}\right| \nrightarrow \infty$, there is a subsequence $\left(\varphi_{j}\right)$ of $\left(X_{n}(\xi)\right)$ and there is a $\varphi \in G$ such that $\varphi_{j} \rightarrow \varphi$ in $G$. Then $\varphi_{j} \omega \rightarrow \varphi \omega$ for all $\omega \in \Omega$. But this implies that $\nu=\delta_{\varphi^{-1} \omega_{0}}$, which contradicts the continuity of $\nu$.

Corollary 2.3. Let $\mu$ be a probability on the free group $\Gamma$ satisfying (1.1). Let $\left(X_{n}\right)$ be the corresponding right random walk on $\Gamma$. Then with probability 1 there is an end $\omega \in \Omega$ such that $X_{n} \rightarrow \omega$ in $\Gamma \cup \Omega$.

Remarks. 1. Corollary 2.3 implies that $\Omega$ is a boundary of $\Gamma$ in the sense of [10]. Furthermore, if $T$ and $\mu$ are as in the Theorem, then $\Omega$ is a boundary of $G$ in the same sense. For we can put a topology on $G \cup \Omega$ making it compact with $G$ as an open dense subset. Indeed, the sets

$$
N_{k}=\left\{\varphi \in G: j\left(\varphi\left(v_{0}\right), \omega\right) \geq k\right\} \cup\left\{\omega^{\prime} \in \Omega: j\left(\omega^{\prime}, \omega\right) \geq k\right\},
$$

for $k=1,2, \ldots$, form a base of neighbourhoods at $\omega \in \Omega$ for such a topology.

2. Let $\mu$ be as in the Theorem or Corollary 2.3. Then there is only one probability $\nu$ on $\Omega$ such that $\mu * \nu=\nu$. For if $Z=\lim _{n \rightarrow \infty} X_{n} \in \Omega$, then $\nu$ must be the distribution of $Z$ (see [10, p. 18]).

\section{Application to Random walks in $\operatorname{PGL}(2, F), F$ a local field}

The general reference for this section is [14, Chapter II]. Let $F$ be a commutative field with a discrete valuation $v$. Let $\pi \in F$ satisfy $v(\pi)=1$, and let $\mathscr{O}=\{x \in F: v(x) \geq 0\}$. Assume that $F$ is complete for the metric associated with $v$ and that the residue field $\mathscr{O} / \pi \mathscr{O}$ has $q<\infty$ elements, so that $F$ is locally compact. Let $G_{0}=\mathrm{GL}(2, F), Z=\left\{\lambda I: \lambda \in F^{\times}\right\}$and $K=\left\{g \in G_{0}: g\right.$ and $g^{-1}$ have entries in $\left.\mathscr{O}\right\}$. Then $\widetilde{G}_{0}=\operatorname{PGL}(2, F)=G_{0} / Z$ is a subgroup of the group $G$ of automorphisms of a homogeneous tree $T$ of degree $q+1$. Indeed, $T$ may be realized as the set of equivalence classes of lattices in the two-dimensional vector space $F^{2}$, where two lattices $L, L^{\prime}$ are equivalent if $L^{\prime}=\lambda L$ for some $\lambda \in F^{\times}$. Let $\left\{e_{1}, e_{2}\right\}$ be the usual basis of $F^{2}$, let $L_{0}=\mathscr{O} e_{1} \oplus \mathscr{O} e_{2}$, and let $v_{0}$ be the class of $L_{0}$. Then $G_{0}$ acts transitively on $T$, and $K Z$ is the stabilizer of $v_{0}$. Thus $T \cong G_{0} / K Z$. The space $\Omega$ of ends of $T$ is isomorphic as a $G_{0}$-space to the set $\mathbf{P}_{1}(F)$ of lines in $F^{2}[14, \mathrm{p} .72]$. The 
stabilizer of $l_{0}=F e_{1} \in \mathbf{P}_{1}(F)$ is $B=\left\{\left(\begin{array}{ll}a & b \\ c & d\end{array}\right) \in G_{0}: c=0\right\}$. So we may identify $\Omega$ with $G_{0} / B$. If $x=\left(\begin{array}{ll}a & b \\ c & d\end{array}\right) Z \in \widetilde{G}_{0}$, then $\left(\begin{array}{l}\bar{a} \\ c\end{array}\right)$ and $\left(\begin{array}{l}\bar{b} \\ d\end{array}\right) \in \mathbf{P}_{1}(F)$ (where $\left.\left(\begin{array}{l}\bar{a} \\ c\end{array}\right)=F\left(a e_{1}+c e_{2}\right)\right)$ are called the columns of $x$. If $\max (|a|,|c|) \geq \max (|b|,|d|)$, where $|t|=q^{-v(t)}$, i.e., if $\min (v(a), v(c)) \leq \min (v(b), v(d))$, we call $\left(\begin{array}{l}\bar{a} \\ c\end{array}\right)$ the larger of the columns of $x$, otherwise we call $\left(\begin{array}{l}\bar{b} \\ d\end{array}\right)$ the larger.

Proposition 3.1. Let $\mu$ be a regular Borel probability on $\widetilde{G}_{0}$. Suppose that $\operatorname{Supp}(\mu)$ is not contained in an amenable subgroup of $\widetilde{G}_{0}$. Let $\left(X_{k}\right)_{k \geq 0}$ be the associated right random walk on $\widetilde{G}_{0}$. Then with probability 1 , the larger of the two columns of $X_{k}$ tends to a limit in $\mathbf{P}_{1}(F)$.

Proof. This will be an immediate consequence of the Theorem once we interpret what it means that $g_{k} K Z \rightarrow g B$ in $T \cup \Omega$. Now $l_{0}$ corresponds to the end $\omega_{0}$ of the chain $\left(v_{0}, v_{1}, v_{2}, \ldots\right)$ in $T$, where $v_{n}$ is the class of the lattice $L_{n}=\mathscr{O} e_{1}+\pi^{n} L_{0}=\mathscr{O} e_{1} \oplus \pi^{n} \mathscr{O} e_{2}=x_{n} L_{0}$ for

$$
x_{n}=\left(\begin{array}{cc}
1 & 0 \\
0 & \pi^{n}
\end{array}\right) \text {. }
$$

For each $g \in G_{0}$ we have $g K Z=g^{\prime} K Z$, where

$$
g^{\prime}=\left(\begin{array}{cc}
1 & 0 \\
\tau & \pi^{\delta}
\end{array}\right) \quad \text { or } \quad g^{\prime}=\left(\begin{array}{cc}
\tau & \pi^{\delta} \\
1 & 0
\end{array}\right)
$$

Here $\delta \geq 0$ is unique and $\tau \in \mathscr{O}$ is unique $\bmod \pi^{\delta}$ within each of the two types in (3.1), and

$$
\left(\begin{array}{cc}
1 & 0 \\
\tau & \pi^{\delta}
\end{array}\right) K Z=\left(\begin{array}{cc}
\tau^{\prime} & \pi^{\delta} \\
1 & 0
\end{array}\right) K Z
$$

can only happen for $\tau=\tau^{\prime}=\delta=0$ or when $\delta>0, v(\tau)=v\left(\tau^{\prime}\right)=0$ and $\tau^{\prime}=\tau^{-1} \bmod \pi^{\delta}$. Also, $\delta=d\left(v_{0}, g v_{0}\right)$. Using this to calculate $d\left(g v_{0}, v_{n}\right)=$ $d\left(v_{0}, g^{-1} x_{n} v_{0}\right)$, we find that $j\left(g v_{0}, \omega\right)$ (measured from $\left.v_{0}\right)$ equals $j\left(g v_{0}, v_{n}\right)$ once $n \geq \delta$, and this equals either $v(\tau), \delta$, or 0 , according as $g^{\prime}$ in (3.1) is of the first type with $\tau \notin \pi^{\delta} \mathscr{O}$, the first type with $\tau \in \pi^{\delta} \mathscr{O}$, or the second type, respectively.

Thus $g_{k} K Z \rightarrow g B$ in $T \cup \Omega$ means that for large $k$,

$$
g^{-1} g_{k} K Z=\left(\begin{array}{cc}
1 & 0 \\
\tau_{k} & \pi^{\delta_{k}}
\end{array}\right) K Z
$$

where $m_{k}=\min \left(v\left(\tau_{k}\right), \delta_{k}\right) \rightarrow \infty$. So if $g=\left(\begin{array}{ll}a & b \\ c & d\end{array}\right)$, we can find $\lambda_{k} \in F^{\times}$ and

$$
\left(\begin{array}{cc}
t_{k} & u_{k} \\
t_{k}^{\prime} & u_{k}^{\prime}
\end{array}\right) \in K
$$


so that

$$
\begin{aligned}
\lambda_{k}^{-1} g_{k} & =\left(\begin{array}{ll}
a & b \\
c & d
\end{array}\right)\left(\begin{array}{cc}
1 & 0 \\
\tau_{k} & \pi^{\delta_{k}}
\end{array}\right)\left(\begin{array}{cc}
t_{k} & u_{k} \\
t_{k}^{\prime} & u_{k}^{\prime}
\end{array}\right) \\
& =\left(\begin{array}{ll}
a t_{k} & a u_{k} \\
c t_{k} & c u_{k}
\end{array}\right) \quad \bmod \pi^{m_{k}+\alpha},
\end{aligned}
$$

where $\alpha=\min (v(b), v(d))$. Since $\min \left(v\left(t_{k}\right), v\left(u_{k}\right)\right)=0$, this proves the result.

\section{REFERENCES}

1. R. Azencott, Espaces de Poisson des groupes localement compacts, Lecture Notes in Mathematics 148, Springer-Verlag, Berlin, Heidelberg, New York, 1970.

2. L. Breiman, Probability, Addison Wesley, Reading, Massachusetts, 1968.

3. J. H. Carruth, J. A. Hildebrant and R. J. Koch, The theory of topological semigroups, Marcel Dekker, New York, Basel, 1983.

4. P. Cartier, Functions harmoniques sur un arbre, Symposia Math. 9 (1972), 203-270.

5. D. I. Cartwright and S. Sawyer, The Martin boundary for general isotropic random walks in a tree, manuscript.

6. Y. Derriennic, Marche aléatoire sur le groupe libre et frontière de Martin, Z. Wahrscheinlichkeitstheorie 32 (1975), 261-276.

7. Y. Derriennic and Y. Guivarch, Théorème de renouvellement pour les groupes non moyennables, C. R. Acad. Sc. Paris Série A 277 (1973), 613-615.

8. E. B. Dynkin and M. B. Malyutov, Random walks on groups with a finite number of generators, Soviet Math. Dokl. 2 (1961), 399-402.

9. H. Furstenberg, Non-commuting random products, Trans. Amer. Math. Soc. 108 (1963), 377428.

10. __ Random walks and discrete subgroups of Lie groups, in Advances in Probability and Related Topics, Vol. I, ed. Peter Ney, Marcel Dekker, New York, 1971, pp. 1-63.

11. V. A. Kaimanovich and A. M. Vershik, Random walks on discrete groups: boundary and entropy, Annals of Prob. 11 (1983), 457-490.

12. C. Nebbia, On the amenability and the Kunze-Stein property for groups acting on a tree, (to appear in Pacific J. Math.).

13. S. Sawyer and T. Steger, The rate of escape for anisotropic random walks in a tree, Probab. Th. Rel. Fields 76 (1987), 207-230.

14. J.-P. Serre, Trees, Springer-Verlag, Berlin, Heidelberg, New York, 1980.

15. V. I. Trofimov, Automorphism groups of graphs as topological objects, Math. Notes 38 (1985), 717-720.

Department of Pure Mathematics, University of Sydney, N. S. W. 2006, Australia

Dipartimento di Matematica, Università di Milano, Via C. Saldini, 50, Milano 20133 , ITALY 